PROCEEDINGS OF THE

AMERICAN MATHEMATICAL SOCIETY

Volume 134, Number 5 , Pages 1503-1512

S 0002-9939(05)08499-6

Article electronically published on December 14, 2005

\title{
SEPARATE CONTINUITY, JOINT CONTINUITY AND THE LINDELÖF PROPERTY
}

\author{
PETAR S. KENDEROV AND WARREN B. MOORS
}

(Communicated by Jonathan M. Borwein)

\begin{abstract}
In this paper we prove a theorem more general than the following. Suppose that $X$ is Lindelöf and $\alpha$-favourable and $Y$ is Lindelöf and Čechcomplete. Then for each separately continuous function $f: X \times Y \rightarrow \mathbb{R}$ there exists a residual set $R$ in $X$ such that $f$ is jointly continuous at each point of $R \times Y$.
\end{abstract}

\section{INTRODUCTION}

If $X, Y$ and $Z$ are topological spaces and $f: X \times Y \rightarrow Z$ is a function, then we say that $f$ is jointly continuous at $\left(x_{0}, y_{0}\right) \in X \times Y$ if for each neighbourhood $W$ of $f\left(x_{0}, y_{0}\right)$ there exists a product of open sets $U \times V \subseteq X \times Y$ containing $\left(x_{0}, y_{0}\right)$ such that $f(U \times V) \subseteq W$ and we say that $f$ is separately continuous on $X \times Y$ if for each $x_{0} \in X$ and $y_{0} \in Y$ the functions $y \mapsto f\left(x_{0}, y\right)$ and $x \mapsto f\left(x, y_{0}\right)$ are both continuous on $Y$ and $X$ respectively. If the range space $Z$ is a metric space with metric $d$, and $\varepsilon$ is a positive number, then we say that $f$ is $\varepsilon$-jointly continuous at $\left(x_{0}, y_{0}\right) \in X \times Y$ if there exists a product of open sets $U \times V \subseteq X \times Y$ containing $\left(x_{0}, y_{0}\right) \in X \times Y$ such that $d$-diam $f(U \times V) \leq \varepsilon$.

Since the paper 1 of Baire first appeared there has been continued interest in the question of when a separately continuous function defined on a product of "nice" spaces admits a point (or many points) of joint continuity and over the years there have been many contributions to this area. Most of these results can be classified into one of two types. (I) The existence problem, i.e., if $f: X \times Y \rightarrow \mathbb{R}$ is separately continuous, find conditions on either $X$ or $Y$ (or both) such that $f$ has at least one point of joint continuity. (II) The fibre problem, i.e., if $f: X \times Y \rightarrow \mathbb{R}$ is separately continuous, find conditions on either $X$ or $Y$ (or both) such that there exists a "big" subset $R$ of $X$ such that $f$ is jointly continuous at the points of $R \times Y$. Our interest in this paper is in the fibre problem. Specifically, we are interested in providing a partial extension of the following result of M. Talagrand, 9, p. 503].

"Let $f: X \times Y \rightarrow \mathbb{R}$ be a separately continuous function defined on the product of Čech-complete spaces $X$ and $Y$. If $Y$ is Lindelöf, then there exists a dense $G_{\delta}$ subset $R$ of $X$ such that $f$ is jointly continuous at each point of $R \times Y$."

Received by the editors July 27, 2004.

2000 Mathematics Subject Classification. Primary 54C05, 22A10; Secondary 54E52, 39B99.

Key words and phrases. Separate continuity, joint continuity, Lindelöf property.

The second author was supported by the Marsden Fund research grant, UOA0422, administered by the Royal Society of New Zealand. 
This result of Talagrand is distinctive within the literature because it does not require the space $Y$ to be either compact ( $\sigma$-compact) or second countable. What we shall do is show that the conclusion of Talagrand's theorem remains valid when one changes the hypothesis on $X$ from being Cech-complete to being Lindelöf and $\alpha$-favourable. Even in the case when $Y$ is compact this seems to be a new result. For more information on problem (II) see [9, pp. 495-536].

Some form of our first lemma may be found in many of the papers written on separate and joint continuity.

Lemma 1. Let $X$ and $Y$ be topological spaces, let $\varepsilon$ be a positive number and let $(Z, d)$ be a metric space. If $f: X \times Y \rightarrow Z$ and $y \mapsto f\left(x_{0}, y\right)$ is continuous on $Y$, but not $\varepsilon$-jointly continuous at $\left(x_{0}, y_{0}\right) \in X \times Y$, then for each pair of open neighbourhoods $U$ of $x_{0}$ and $V$ of $y_{0}$ there exist points $x$ and $x^{\prime}$ in $U$ and $y$ in $V$ such that $\varepsilon / 3<d\left(f(x, y), f\left(x^{\prime}, y\right)\right)$.

Proof. Let $U \times V \subseteq X \times Y$ be a product of open sets containing the point $\left(x_{0}, y_{0}\right) \in$ $X \times Y$. Since $y \mapsto f\left(x_{0}, y\right)$ is continuous on $Y$ we can assume, by possibly making $V$ smaller, that $d\left(f\left(x_{0}, y\right), f\left(x_{0}, y_{0}\right)\right)<\varepsilon / 6$ for all $y \in V$. Since $f$ is not $\varepsilon$-jointly continuous at $\left(x_{0}, y_{0}\right)$ there exist points $(x, y)$ and $\left(x^{\prime}, y^{\prime}\right)$ in $U \times V$ such that $\varepsilon<d\left(f(x, y), f\left(x^{\prime}, y^{\prime}\right)\right)$. On the other hand,

$$
\begin{aligned}
d\left(f(x, y), f\left(x^{\prime}, y^{\prime}\right)\right) \leq & d\left(f(x, y), f\left(x_{0}, y\right)\right)+d\left(f\left(x_{0}, y\right), f\left(x_{0}, y^{\prime}\right)\right) \\
& +d\left(f\left(x_{0}, y^{\prime}\right), f\left(x^{\prime}, y^{\prime}\right)\right) \\
& <d\left(f(x, y), f\left(x_{0}, y\right)\right)+d\left(f\left(x_{0}, y^{\prime}\right), f\left(x^{\prime}, y^{\prime}\right)\right)+\varepsilon / 3 .
\end{aligned}
$$

Therefore, either $\varepsilon / 3<d\left(f(x, y), f\left(x_{0}, y\right)\right)$ or $\varepsilon / 3<d\left(f\left(x_{0}, y^{\prime}\right), f\left(x^{\prime}, y^{\prime}\right)\right)$.

For a topological space $Y$ we shall denote by $C(Y)$ the set of all real-valued continuous functions defined on $Y$ and by $C_{p}(Y)$ the set $C(Y)$ endowed with the topology of pointwise convergence on $Y$. Further, if $X$ is a topological space and $f: X \rightarrow C(Y)$, then the mapping $\tilde{f}: X \times Y \rightarrow \mathbb{R}$ defined by $\tilde{f}(x, y):=f(x)(y)$ is separately continuous on $X \times Y$ if and only if $f: X \rightarrow C_{p}(Y)$ is continuous. Hence, there is a natural correspondence between the study of real-valued separately continuous functions on $X \times Y$ and the study of continuous mappings from $X$ into $C_{p}(Y)$. With this in mind, we introduce the following definitions. We say that a mapping $f: X \rightarrow C(Y)$ is jointly continuous at $\left(x_{0}, y_{0}\right) \in X \times Y$ if the function $\tilde{f}$ is jointly continuous at $\left(x_{0}, y_{0}\right)$ and, for each $\varepsilon>0$, we will say that $f$ is $\varepsilon$-jointly continuous at $\left(x_{0}, y_{0}\right)$ if the function $\tilde{f}$ is $\varepsilon$-jointly continuous at $\left(x_{0}, y_{0}\right)$.

With these definitions under our belt we can rephrase Lemma 1 as follows.

Lemma 2. Let $X$ and $Y$ be topological spaces and let $f: X \rightarrow C(Y)$. If, for some $\varepsilon>0, f$ is not $\varepsilon$-jointly continuous at $\left(x_{0}, y_{0}\right) \in X \times Y$, then for each pair of open neighbourhoods $U$ of $x_{0}$ and $V$ of $y_{0}$ there exist points $x$ and $x^{\prime}$ in $U$ and $y$ in $V$ such that $\varepsilon / 3<f(x)(y)-f\left(x^{\prime}\right)(y)$.

In addition to the previously mentioned notions of continuity, we shall also require a weaker form of continuity. If $f: X \rightarrow Y$ is a function acting from a topological space $X$ into a topological, $Y$ space then we say that $f$ is quasi-continuous on $X$ if for each pair of open subsets $U$ of $X$ and $W$ of $Y$ such that $f(U) \cap W \neq \emptyset$, there exists a non-empty open set $V \subseteq U$ such that $f(V) \subseteq W$. Although in general quasi-continuous functions are not obliged to have any points of continuity, the next 
lemma shows that when the domain space is Baire and the range space is metric such mappings must have many points of continuity.

Lemma 3 (2]). Let $f: X \rightarrow Y$ be a quasi-continuous mapping acting from a Baire space $X$ into a metric space $Y$. Then $\{x \in X: f$ is continuous at $x\}$ is residual in $X$ (i.e., contains a countable intersection of dense open subsets of $X$ ).

For more information on the continuity of quasi-continuous mappings see [6].

\section{Main Result}

The main result of this paper (Theorem 1) is based upon Lemma 6 which in turn is based upon the following lemma.

Lemma 4 ([3, Corollary $\mathrm{C}])$. Let $Y$ be a compact and $D$ a dense and countable subset of $Y$. Then every subset of $C(Y)$ which is compact with respect to the topology of pointwise convergence on $D$ and Lindelof with respect to the topology of pointwise convergence on $Y$ is separable in $\left(C(Y),\|\cdot\|_{\infty}\right)$.

The proof of Lemma 6 relies upon the careful handling of second category sets (i.e., sets that are not of the first category). Here we shall introduce some notation that will facilitate this. Let $X$ be a topological space and let $U$ be an open subset of $X$. We say that a subset $A$ of $X$ is everywhere second category in $U$ if $A \cap W$ is second category in $X$ for each non-empty open subset $W$ of $U$. For a subset $A$ of $X$ we shall denote by $D(A)$ the union of all open subsets $W$ for which $A$ is everywhere second category in $W$. It is readily seen that $A$ is everywhere second category in $D(A)$ (i.e., $D(A)$ is the largest open subset of $X$ in which $A$ is everywhere second category). Although in general $D(A)$ may be empty, it follows from 4, Proposition $3.2 .5]$ that if $A$ is second category in $X$, then $D(A) \neq \emptyset$.

Lemma 6 also requires a version of the pigeonhole principle.

Lemma 5 (Pigeonhole principle for second category sets). Let $f: X \rightarrow Y$ be a mapping from a second category set $X$ into a non-empty set $Y$. If $\left(V_{n}\right)_{n \in \mathbb{N}}$ is a cover of $Y$, then for at least one $n \in \mathbb{N}, f^{-1}\left(V_{n}\right)$ is second category in $X$.

Lemma 6. Let $Y$ be a Lindelöf Čech-complete space, let $(X, d)$ be a complete metric space and let $f: X \rightarrow C_{p}(Y)$ be a quasi-continuous mapping. If there exists a Lindelöf subspace $L$ of $C_{p}(Y)$ such that $f(X) \subseteq L$, then there exists a residual subset $R$ of $X$ such that $f$ is jointly continuous at each point of $R \times Y$.

Proof. Let $\beta Y$ denote the Stone-Čech-compactification of $Y$ and let $\left(G_{n}\right)_{n \geq 0}$ be a decreasing sequence of open subsets of $\beta Y$ such that: (i) $G_{0}:=\beta Y$ and (ii) $Y=\bigcap_{n \geq 0} G_{n}$. For each $\varepsilon>0$ consider the set

$$
R_{\varepsilon}:=\{x \in X: f \text { is } \varepsilon \text {-jointly continuous at each point of }\{x\} \times Y\} .
$$

Clearly, $f$ is jointly continuous at each point of $\left(\bigcap_{n \in \mathbb{N}} R_{1 / n}\right) \times Y$. Therefore, it will be sufficient to show that for each $\varepsilon>0, R_{\varepsilon}$ is residual in $X$. To this end, let us fix $\varepsilon>0$. In order to obtain a contradiction let us assume that $X \backslash R_{\varepsilon}$ is second category in $X$. (Note that for each $x \in X \backslash R_{\varepsilon}$ there exists an element $y \in Y$ such that $f$ is not $\varepsilon$-jointly continuous at $(x, y)$.) Let $T$ be the set of all finite sequences of 0's and 1's. We shall inductively (on the length $|t|$ of $t \in T$ ) define the following: second category subsets $X_{t}$ of $X \backslash R_{\varepsilon}$; points $x_{t}$ and $x_{t}^{\prime}$ in $D\left(X_{t}\right)$; non-empty open subsets $Y_{t}$ of $\beta Y$; elements $y_{t} \in Y_{t} \cap Y$ and sequences $\left(O_{n}^{t}\right)_{n \in \mathbb{N}}$ of dense open subsets of $X$ that fulfill the following properties. 
(i) $X_{t} \subseteq X_{t^{\prime}}$ and $Y_{t} \subseteq Y_{t^{\prime}}$ whenever $t^{\prime}<t$ (i.e., whenever $t$ is an extension of $\left.t^{\prime}\right)$

(ii) $d$-diam $\left(X_{t}\right)<1 / 2^{|t|}$ and $\bar{Y}_{t}^{\beta Y} \subseteq G_{|t|}$, where $\bar{Y}_{t}^{\beta Y}$ denotes the closure of $Y_{t}$ in $\beta Y$;

(iii) $\overline{X_{t 0}} \cap \overline{X_{t 1}}=\emptyset$;

(iv) for each $x \in X_{t}$ there exists a $y_{x} \in Y_{t} \cap Y$ such that $f$ is not $\varepsilon$-jointly continuous at $\left(x, y_{x}\right)$;

(v) $\varepsilon / 3<f\left(x_{t}\right)\left(y_{t}\right)-f\left(x_{t}^{\prime}\right)\left(y_{t}\right)$;

(vi) $\varepsilon / 3<f(x)\left(y_{t}\right)-f\left(x^{\prime}\right)\left(y_{t}\right)$ for all $x \in \bar{X}_{t 1}$ and all $x^{\prime} \in \bar{X}_{t 0}$;

(vii) the mapping $x \mapsto f(x)\left(y_{t}\right)$ is continuous at the points of $\bigcap_{n \in \mathbb{N}} O_{n}^{t}$;

(viii) if $\left|t^{\prime}\right|<|t|$, then $\overline{X_{t}} \subseteq \bigcap\left\{O_{k}^{t^{\prime}}: 1 \leq k \leq|t|\right\}$.

Base step. Let $X_{\emptyset}$ be any second category subset of $X \backslash R_{\varepsilon}$ with $d$-diameter less than 1 such that $X_{\emptyset} \subseteq D\left(X_{\emptyset}\right)$. (Note: such a subset exists. For example, if $W$ is any non-empty open subset of $D\left(X \backslash R_{\varepsilon}\right)$ of $d$-diameter less than 1 , then one could set $X_{\emptyset}:=W \cap\left(X \backslash R_{\varepsilon}\right)$.) Let $Y_{\emptyset}:=\beta Y$. Since $X_{\emptyset}$ is a subset of $X \backslash R_{\varepsilon}$, for every $x \in X_{\emptyset}$ there exists a $y_{x} \in Y_{\emptyset} \cap Y$ such that $f$ is not $\varepsilon$-jointly continuous at $\left(x, y_{x}\right)$. Therefore, by Lemma 2 there exist points $x_{\emptyset}$ and $x_{\emptyset}^{\prime}$ in $D\left(X_{\emptyset}\right)$ and an element $y_{\emptyset} \in Y_{\emptyset} \cap Y$ such that

$$
\varepsilon / 3<f\left(x_{\emptyset}\right)\left(y_{\emptyset}\right)-f\left(x_{\emptyset}^{\prime}\right)\left(y_{\emptyset}\right) .
$$

Also since the mapping $x \mapsto f(x)\left(y_{\emptyset}\right)$ is quasi-continuous it follows from Lemma 3 that there exists a sequence $\left(O_{n}^{\emptyset}\right)_{n \in \mathbb{N}}$ of dense open subsets of $X$ such that the mapping $x \mapsto f(x)\left(y_{\emptyset}\right)$ is continuous at the points of $\bigcap_{n \in \mathbb{N}} O_{n}^{\emptyset}$.

Assuming that we have defined the second category subsets $X_{t}$ of $X$, the points $x_{t}$ and $x_{t}^{\prime} \in D\left(X_{t}\right)$, the non-empty open subsets $Y_{t}$ of $\beta Y$, the elements $y_{t} \in Y_{t} \cap Y$ and the sequences $\left(O_{n}^{t}\right)_{n \in \mathbb{N}}$ of dense open subsets of $X$ that satisfy the properties (i)-(viii) for each $t \in T$ with $|t| \leq n$, we shall proceed to the next step.

Inductive step. Consider $t \in T$ of length $n$. Since $\varepsilon / 3<f\left(x_{t}\right)\left(y_{t}\right)-f\left(x_{t}^{\prime}\right)\left(y_{t}\right)$ and $x \mapsto f(x)\left(y_{t}\right)$ is quasi-continuous there exist non-empty open sets $W_{0}$ and $W_{1}$ with $d$-diameter less than $1 / 2^{n+1}$ such that

$$
\emptyset=\overline{W_{0}} \cap \overline{W_{1}} \subseteq \overline{W_{0}} \cup \overline{W_{1}} \subseteq D\left(X_{t}\right) \cap \bigcap\left\{O_{k}^{t^{\prime}}: 1 \leq k \leq n+1 \text { and }\left|t^{\prime}\right|<n+1\right\}
$$

and $\varepsilon / 3<f(x)\left(y_{t}\right)-f\left(x^{\prime}\right)\left(y_{t}\right)$ for all $x \in \overline{W_{1}}$ and all $x^{\prime} \in \overline{W_{0}}$. Now, since $\overline{Y_{t} \cap Y^{Y}}$ (i.e., the closure of $Y_{t} \cap Y$ with respect to $Y$ ) is Lindelöf, there exist open subsets $\left(V_{n}\right)_{n \in \mathbb{N}}$ of $\beta Y$ such that

$$
Y_{t} \cap Y \subseteq{\overline{Y_{t} \cap Y}}^{Y} \subseteq \bigcup_{n \in \mathbb{N}} V_{n} \subseteq \bigcup_{n \in \mathbb{N}}{\overline{V_{n}}}^{\beta Y} \subseteq G_{n+1}
$$

Hence, by Lemma 5 , for each $i \in\{0,1\}$ there exists a second category subset $X_{t i} \subseteq$ $X_{t} \cap W_{i}$ and an $n_{i} \in \mathbb{N}$ such that for each $x \in X_{t i}$ there exists a $y_{x} \in V_{n_{i}} \cap Y_{t} \cap Y$ for which $f$ is not $\varepsilon$-jointly continuous at $\left(x, y_{x}\right)$. Therefore, by Lemma 2 , for each $i \in\{0,1\}$ there exist points $x_{t i}$ and $x_{t i}^{\prime}$ in $D\left(X_{t i}\right)$ and an element $y_{t i} \in V_{n_{i}} \cap Y_{t} \cap Y$ such that $\varepsilon / 3<f\left(x_{t i}\right)\left(y_{t i}\right)-f\left(x_{t i}^{\prime}\right)\left(y_{t i}\right)$. Also since the mapping $x \mapsto f(x)\left(y_{t i}\right)$ is quasi-continuous it follows from Lemma 3 that there exists a sequence $\left(O_{n}^{t i}\right)_{n \in \mathbb{N}}$ of dense open subsets of $X$ such that the mapping $x \mapsto f(x)\left(y_{t i}\right)$ is continuous at the points of $\bigcap_{n \in \mathbb{N}} O_{n}^{t i}$. The inductive step is completed by defining $Y_{t i}:=V_{n_{i}} \cap Y_{t}$. 
Let $E:=\left\{y_{t}: t \in T\right\}$. We claim that $\bar{E}^{Y}$ (i.e., the closure of $E$ with respect to $Y)$ is a compact subset of $Y$. To see this, note that for each $n \in \mathbb{N}$

$$
\bar{E}^{\beta Y} \subseteq \bigcup_{|t|=n} \bar{Y}_{t}^{\beta Y} \cup\left\{y_{t}: 0 \leq|t|<n\right\} \subseteq \bigcup_{|t|=n} \bar{Y}_{t}^{\beta Y} \cup E \subseteq \bigcup_{|t|=n} \bar{Y}_{t}^{\beta Y} \cup Y \subseteq G_{n}
$$

and so $\bar{E}^{\beta Y} \subseteq \bigcap_{n \geq 0} G_{n}=Y$. Therefore, $\bar{E}^{Y}=\bar{E}^{\beta Y}$, which is compact in $\beta Y$.

By the construction, the set $K:=\bigcap_{n \in \mathbb{N}} K_{n}$, where $K_{n}:=\bigcup_{|t|=n} \overline{X_{t}}$, is a closed and totally bounded subset of $X$ (and hence compact, since $X$ is complete). Furthermore, the construction also yields that for each $t \in T, K \subseteq \bigcap_{n \in \mathbb{N}} O_{n}^{t}$. Thus, for each $t \in T$, the mapping $x \mapsto f(x)\left(y_{t}\right)$ is continuous on $K$. Note also that for each pair of distinct points $x$ and $x^{\prime}$ in $K$ there exists a $t \in T$ such that $\varepsilon / 3<\left|f(x)\left(y_{t}\right)-f\left(x^{\prime}\right)\left(y_{t}\right)\right|$. Next, we consider the continuous mapping $\mathscr{R}: C_{p}(Y) \rightarrow C_{p}\left(\bar{E}^{Y}\right)$ defined by $\mathscr{R}(f):=\left.f\right|_{\bar{E}^{Y}}$. Then $(\mathscr{R} \circ f)(K)$ is a nonseparable subset of $\left(C\left(\bar{E}^{Y}\right),\|\cdot\|_{\infty}\right)$ that is compact with respect to the topology of pointwise convergence on $E$. Moreover, since the topology of pointwise convergence on $E$ is Hausdorff in $C\left(\bar{E}^{Y}\right),(\mathscr{R} \circ f)(K)$ is closed in this topology and hence closed in $C_{p}\left(\bar{E}^{Y}\right)$. However, by Lemma 4 , this is impossible since $(\mathscr{R} \circ f)(K) \subseteq \mathscr{R}(L)$; which is Lindelöf. Hence, it must be the case that for each $\varepsilon>0, R_{\varepsilon}$ is residual in $X$.

To formulate the statement of our main theorem we will need to consider the following topological game.

Let $X$ be a topological space. On $X$ we shall consider the Choquet game played between two players $\alpha$ and $\beta$. A play of this game is a decreasing sequence of, alternately chosen, non-empty open subsets $A_{n} \subseteq B_{n} \subseteq \ldots \subseteq B_{2} \subseteq A_{1} \subseteq B_{1}$, where the sets $A_{n}$ are chosen by player $\alpha$ and the sets $B_{n}$ by player $\beta$. The player $\alpha$ is said to have won a play of the Choquet game if $\bigcap_{n \in N} B_{n} \neq \emptyset$. Otherwise, player $\beta$ is said to have won the play. A strategy $s$ for the player $\alpha$ is a rule that tells him or her how to play (possibly depending on all the previous moves of player $\beta$ ). Since the moves of player $\alpha$ may depend on the previous moves of player $\beta$, we denote the $n^{\text {th }}$ move of player $\alpha$ by $s\left(B_{1}, B_{2}, \ldots, B_{n}\right)$. Any sequence of non-empty open subsets $\left(B_{n}\right)_{n \in \mathbb{N}}$ of $X$ that satisfy $B_{n+1} \subseteq s\left(B_{1}, B_{2}, \ldots, B_{n}\right)$ for all $n \in \mathbb{N}$ is called an $s$-play. We say that $s$ is a winning strategy if, using it, player $\alpha$ wins every play, independently of the moves of player $\beta$ (i.e., $\bigcap_{n \in \mathbb{N}} B_{n} \neq \emptyset$ for each $s$-play $\left.\left(B_{n}\right)_{n \in \mathbb{N}}\right)$. A topological space $X$ is called an $\alpha$-favourable space if $\alpha$ has a winning strategy in the Choquet game played on $X$. More information on the Choquet game can be found in [13].

Let $X$ be an $\alpha$-favourable space and let $s$ be a winning strategy for the player $\alpha$ in the Choquet game played on $X$. We shall denote by $P$ the space of all $s$-plays endowed with the Baire metric $d$, that is, if $p:=\left(B_{n}\right)_{n \in \mathbb{N}}$ and $p^{\prime}:=\left(B_{n}^{\prime}\right)_{n \in \mathbb{N}}$, then $d\left(p, p^{\prime}\right):=0$ if $p=p^{\prime}$ and $d\left(p, p^{\prime}\right):=1 / n$; otherwise, where $n:=\min \{i \in$ $\left.\mathbb{N}: B_{i} \neq B_{i}^{\prime}\right\}$. It is straightforward to verify that $(P, d)$ is a complete metric space, [6]. In the proofs of Lemma 7 and Theorem 1 we shall use the notation $I(p, m):=s\left(B_{1}, B_{2}, \ldots, B_{m}\right)$ for each $m \in \mathbb{N}$ and $p:=\left(B_{n}\right)_{n \in \mathbb{N}} \in P$. Note also that the proofs of Lemma 7 and Theorem 1 are based upon ideas from [8]. 
Lemma 7. Let $X$ be an $\alpha$-favourable space, let $s$ be a winning strategy for the player $\alpha$ in the Choquet game played on $X$ and let $P$ denote the space of all $s$ plays, endowed with the Baire metric. If $f: X \rightarrow Z$ is a quasi-continuous mapping into a topological space $Z$, then the set-valued mapping $F: P \rightarrow 2^{Z}$ is defined by

$$
F(p):=\bigcap_{n \in \mathbb{N}} f\left(B_{n}\right), \quad \text { where } p:=\left(B_{n}\right)_{n \in \mathbb{N}} \text { is an s-play, }
$$

has non-empty values and is a "minimal mapping" (i.e., for every pair of open subsets $U$ of $P$ and $W$ of $Z$ such that $F(U) \cap W \neq \emptyset$ there exists a non-empty open subset $V \subseteq U$ such that $F(V) \subseteq W)$.

Proof. The fact that $F$ has non-empty images follows directly from the observation that for each $p:=\left(B_{n}\right)_{n \in \mathbb{N}} \in P$,

$$
\emptyset \neq f\left(\bigcap_{n \in \mathbb{N}} B_{n}\right) \subseteq \bigcap_{n \in \mathbb{N}} f\left(B_{n}\right)=F(p) .
$$

Next we deduce the minimality of $F$. To this end, let $U$ be an open subset of $P$ and let $W$ be an open subset of $Z$ such that $F(U) \cap W \neq \emptyset$. Choose $p:=\left(B_{n}\right)_{n \in \mathbb{N}} \in U$ and $m \in \mathbb{N}$ such that $F(p) \cap W \neq \emptyset$ and $B(p ; 1 / m)=\left\{p^{\prime} \in P:\left.p^{\prime}\right|_{m}=\left.p\right|_{m}\right\} \subseteq U$. Then $f(I(p, m)) \cap W \neq \emptyset$. Since $f$ is quasi-continuous there exists a non-empty open subset $V^{\prime}$ of $I(p, m)$ such that $f\left(V^{\prime}\right) \subseteq W$. Now we define $p^{*}:=\left(B_{n}^{*}\right)_{n \in \mathbb{N}} \in P$ by $B_{k}^{*}:=B_{k}$ for all $1 \leq k \leq m, B_{m+1}^{*}:=V$ and $B_{k+1}^{*}:=s\left(B_{1}^{*}, \ldots, B_{k}^{*}\right)$ for all $k>m$. Note that this definition is well-defined since

$$
B_{m+1}^{*}=V \subseteq I(p, m)=s\left(B_{1}, \ldots, B_{m}\right)=s\left(B_{1}^{*}, \ldots, B_{m}^{*}\right) .
$$

Then for every $p^{\prime} \in V:=B\left(p^{*} ; 1 /(m+1)\right)$

$$
F\left(p^{\prime}\right)=\bigcap_{n \in \mathbb{N}} f\left(I\left(p^{\prime}, n\right)\right) \subseteq f\left(I\left(p^{\prime}, m+1\right)\right)=f\left(I\left(p^{*}, m+1\right)\right) \subseteq f(V) \subseteq W .
$$

To exploit the previous lemma we need to establish the connection between minimal mappings and the continuity of their selections.

Lemma 8 ([11, Lemma 1.1]). Let $F: X \rightarrow 2^{Z}$ be a minimal mapping acting from a topological space $X$ into non-empty subsets of a Hausdorff space $Z$ and let $\sigma: X \rightarrow Z$ be any selection of $F$ (i.e., $\sigma(x) \in F(x)$ for all $x \in X)$. If $\sigma$ is continuous at $x_{0} \in X$, then $F\left(x_{0}\right)=\left\{\sigma\left(x_{0}\right)\right\}$.

Theorem 1. Let $Y$ be a Lindelöf $\check{C}$ ech-complete space, let $X$ be an $\alpha$-favourable space and let $f: X \rightarrow C_{p}(Y)$ be a quasi-continuous mapping. If there exists a Lindelöf subspace $L$ of $C_{p}(Y)$ such that $f(X) \subseteq L$, then there exists a residual subset $R$ of $X$ such that $f$ is jointly continuous at each point of $R \times Y$.

Proof. Let $s$ be a winning strategy for the player $\alpha$ in the Choquet game played on $X$ and let $P$ denote the space of all $s$-plays, endowed with the Baire metric $d$. On the complete metric space $(P, d)$ we define a set-valued mapping $F: P \rightarrow 2^{C(Y)}$ by

$$
F(p):=\bigcap_{n \in \mathbb{N}} f\left(B_{n}\right), \quad \text { where } p:=\left(B_{n}\right)_{n \in \mathbb{N}} \text { is an } s \text {-play. }
$$

By Lemma 7, the mapping $F$ has non-empty values and is a minimal mapping. Let $\sigma: P \rightarrow C(Y)$ be any selection of $F$. Then since $F$ is minimal, $\sigma$ is quasicontinuous. Hence, by Lemma 6 , there exists a sequence $\left(O_{n}\right)_{n \in \mathbb{N}}$ of dense open 
subsets of $P$ such that $\sigma$ is jointly continuous at each point of $\left(\bigcap_{n \in \mathbb{N}} O_{n}\right) \times Y$. Note that, in particular, this means that $\sigma$ is continuous on $R^{\prime}:=\bigcap_{n \in \mathbb{N}} O_{n}$ when $C(Y)$ is considered with the topology of pointwise convergence on $Y$. Therefore, by Lemma $8, F(p)=\{\sigma(p)\}$ for each $p \in R^{\prime}$. We shall now show that there exists a residual subset $R$ of $X$ and a continuous mapping $g: R \rightarrow R^{\prime}$ such that $f(x)=\sigma(g(x))$ for each $x \in R$. To achieve this, we shall inductively construct a sequence $\left(\Lambda^{n}: n \in \mathbb{N}\right)$ of subsets of $P \times \mathbb{N}$.

Step 0 . Let $\Lambda^{0}$ be a maximal, with respect to set inclusion, subset of $P \times\{1\}$ such that $\left\{I(p, m):(p, m) \in \Lambda^{0}\right\}$ is disjoint. By Zorn's lemma such a maximal family exists. Moreover, it is easy to see that for such a family, $W^{0}:=\bigcup\{I(p, m)$ : $\left.(p, m) \in \Lambda^{0}\right\}$ is dense in $X$.

For each $n \in \mathbb{N}$, we will require the subset $\Lambda^{n}$ of $P \times \mathbb{N}$ to possess the following properties:

$\left(a_{n}\right)\left\{I(p, m):(p, m) \in \Lambda^{n}\right\}$ is a disjoint family of subsets of $X$;

$\left(b_{n}\right) W^{n}:=\bigcup\left\{I(p, m):(p, m) \in \Lambda^{n}\right\}$ is dense in $X$;

$\left(c_{n}\right)\left\{p^{\prime} \in P:\left.p^{\prime}\right|_{m}=\left.p\right|_{m}\right\}=B(p ; 1 / m) \subseteq O_{n}$ for each $(p, m) \in \Lambda^{n}$;

$\left(d_{n}\right)$ for each $(p, m) \in \Lambda^{n}$ there exists a unique $\left(p^{\prime}, m^{\prime}\right) \in \Lambda^{n-1}$ such that $m^{\prime}<m$ and $\left.p^{\prime}\right|_{m^{\prime}}=\left.p\right|_{m^{\prime}}$.

Step 1. Consider $\Lambda^{1} \subseteq P \times \mathbb{N}$ satisfying the properties $\left(a_{1}\right),\left(c_{1}\right)$ and $\left(d_{1}\right)$ and which is maximal with respect to set inclusion. By Zorn's lemma such a maximal family exists. We shall show that $\Lambda^{1}$ satisfies $\left(b_{1}\right)$. If $W^{1}:=\bigcup\{I(p, m):(p, m) \in$ $\left.\Lambda^{1}\right\}$ is not dense in $X$, then there exists a non-empty open subset $U$ of $X$ such that $W^{1} \cap U=\emptyset$. Since $W^{0}$ is dense in $X, W^{0} \cap U \neq \emptyset$ and so we may assume that $U \subseteq I(p, m)$ for some $(p, m) \in \Lambda^{0}$, with $p:=\left(B_{k}\right)_{k \in \mathbb{N}}$ and $m=1$. Now we define $p^{*}:=\left(B_{k}^{*}\right)_{k \in \mathbb{N}} \in P$ by $B_{1}^{*}:=B_{1}, B_{2}^{*}:=U$ and $B_{k+1}^{*}:=s\left(B_{1}^{*}, \ldots, B_{k}^{*}\right)$ for all $k>1$. Note that this defines a legitimate $s$-play since

$$
B_{2}^{*}=U \subseteq I(p, m)=I(p, 1)=s\left(B_{1}\right)=s\left(B_{1}^{*}\right) .
$$

Next, choose $p^{* *} \in B\left(p^{*} ; 1 / 2\right) \cap O_{1} \neq \emptyset$ and $m^{* *} \in \mathbb{N}$ so that $1<m^{* *}$ and $B\left(p^{* *} ; 1 / m^{* *}\right) \subseteq B\left(p^{*} ; 1 / 2\right) \cap O_{1}$. Note: this choice is possible too, since $O_{1}$ is dense and open in $P$. Now, $\left(p^{* *}, m^{* *}\right) \notin \Lambda^{1}$ and $\Lambda^{1} \cup\left\{\left(p^{* *}, m^{* *}\right)\right\}$ is a subset of $P \times \mathbb{N}$ satisfying $\left(a_{1}\right),\left(c_{1}\right)$ and $\left(d_{1}\right)$. This contradicts the maximality of $\Lambda^{1}$ and hence we may conclude that $\Lambda^{1}$ satisfies property $\left(b_{1}\right)$.

Assuming that we have constructed the subsets $\Lambda^{k}$ in the sequence satisfying the properties $\left(a_{k}\right),\left(b_{k}\right),\left(c_{k}\right)$ and $\left(d_{k}\right)$ up to and including the $n^{\text {th }}$ step, we proceed to construct the next step.

Step $(n+1)$. Consider $\Lambda^{n+1} \subseteq P \times \mathbb{N}$ satisfying the properties $\left(a_{n+1}\right),\left(c_{n+1}\right)$ and $\left(d_{n+1}\right)$ and which is maximal with respect to set inclusion. We shall show that $\Lambda^{n+1}$ satisfies property $\left(b_{n+1}\right)$. If $W^{n+1}:=\bigcup\left\{I(p, m):(p, m) \in \Lambda^{n+1}\right\}$ is not dense in $X$, then there exists a non-empty open subset $U$ of $X$ such that $W^{n+1} \cap U=\emptyset$. Since $W^{n}$ is dense in $X, W^{n} \cap U \neq \emptyset$ and so we may assume that $U \subseteq I(p, m)$ for some $(p, m) \in \Lambda^{n}$, with $p:=\left(B_{k}\right)_{k \in \mathbb{N}}$. Now we define $p^{*}:=\left(B_{k}^{*}\right)_{k \in \mathbb{N}} \in P$ by $B_{k}^{*}:=B_{k}$ for all $1 \leq k \leq m, B_{m+1}^{*}=U$ and $B_{k+1}^{*}:=s\left(B_{1}^{*}, \ldots, B_{k}^{*}\right)$ for all $k>m$. Note that this defines a legitimate $s$-play since

$$
B_{m+1}^{*}=U \subseteq I(p, m)=s\left(B_{1}, \ldots, B_{m}\right)=s\left(B_{1}^{*}, \ldots, B_{m}^{*}\right) .
$$

Next, choose $p^{* *} \in B\left(p^{*} ; 1 /(m+1)\right) \cap O_{n+1} \neq \emptyset$ and $m^{* *} \in \mathbb{N}$ so that $m<m^{* *}$ and $B\left(p^{* *} ; 1 / m^{* *}\right) \subseteq B\left(p^{*} ; 1 /(m+1)\right) \cap O_{n+1}$. Note: this choice is possible too, since $O_{n+1}$ is dense and open in $P$. Now, $\left(p^{* *}, m^{* *}\right) \notin \Lambda^{n+1}$ and $\Lambda^{n+1} \cup\left\{\left(p^{* *}, m^{* *}\right)\right\}$ 
is a subset of $P \times \mathbb{N}$ satisfying $\left(a_{n+1}\right),\left(c_{n+1}\right)$ and $\left(d_{n+1}\right)$. This contradicts the maximality of $\Lambda^{n+1}$ and hence we may conclude that $\Lambda^{n+1}$ satisfies property $\left(b_{n+1}\right)$. This completes the inductive step.

Set $R:=\bigcap_{n \in \mathbb{N}} W^{n}$. Then each $x \in R$ uniquely determines a sequence $\left(p^{k}, m^{k}\right) \in$ $\Lambda^{k}, k=1,2, \ldots$, so that $x \in I\left(p^{k}, m^{k}\right)$ and the properties $\left(a_{k}\right)-\left(d_{k}\right)$ are fulfilled. In particular, for every $n, n^{\prime} \in \mathbb{N}, p^{n+n^{\prime}}$ is a continuation of $\left.p^{n}\right|_{m^{n}}$ and, therefore, $d\left(p^{n}, p^{n+n^{\prime}}\right)<1 / m^{n}$. Since $P$ is a complete metric space there exists $p^{\infty}(x):=$ $\lim _{n \rightarrow \infty} p^{n}$ in $P$ which is a continuation of all the $\left.p^{k}\right|_{m^{k}}, k=1,2, \ldots$ Thus, by $\left(c_{n}\right), p^{\infty}(x) \in \bigcap_{k \in \mathbb{N}} O_{k}=R^{\prime}$. Define $g: R \rightarrow R^{\prime}$ by $g(x):=p^{\infty}(x)$. This mapping is continuous on its domain $R$ because, for every pair $x^{\prime}, x^{\prime \prime} \in R \cap I(p, m)$ with $(p, m) \in \Lambda_{k}$, both $g\left(x^{\prime}\right)$ and $g\left(x^{\prime \prime}\right)$ are continuations of $\left.p\right|_{m}$ (i.e., $d\left(g\left(x^{\prime}\right), g\left(x^{\prime \prime}\right)\right)<$ $1 / m)$. Further, since $I\left(p^{\infty}(x), m^{k}\right)=I\left(p^{k}, m^{k}\right)$ for all $k \in \mathbb{N}$ we may deduce that

$$
\begin{aligned}
f(x) \in f\left(\bigcap_{k \in \mathbb{N}} I\left(p^{k}, m^{k}\right)\right) \subseteq \bigcap_{k \in \mathbb{N}} f\left(I\left(p^{k}, m^{k}\right)\right)=\bigcap_{k \in \mathbb{N}} f\left(I\left(p^{\infty}(x), m^{k}\right)\right) \\
=\bigcap_{k \in \mathbb{N}} f\left(I\left(p^{\infty}(x), k\right)\right)=F\left(p^{\infty}(x)\right)=F(g(x))=\{\sigma(g(x))\} ;
\end{aligned}
$$

which shows that $f(x)=\sigma(g(x))$ for all $x \in R$.

Thus, it follows that $\left.f\right|_{R}$ is jointly continuous at each point of $R$. Then with a small amount of extra effort we can deduce that $f$ is in fact jointly continuous at each point of $R$.

Remark. If one really wanted to "squeeze the pips" out of the previous theorem, one could prove the slightly more general statement given below.

"Let $Y$ be a Lindelöf Čech-complete space, let $D \subseteq Y$ be a dense subset, let $X$ be an $\alpha$-favourable space and let $f: X \rightarrow C(Y)$ be a quasi-continuous mapping with respect to the topology of pointwise convergence on $D$. If $\left\{\left.f(x)\right|_{E}: x \in X\right\}$ is contained in a Lindelöf subset of $C_{p}(\bar{E})$ for each countable subset $E$ of $D$ that is relatively compact in $Y$, then there exists a residual subset $R$ of $X$ such that $f$ is jointly continuous at each point of $R \times Y$."

To prove this more general statement we first need to improve Lemma 1 and correspondingly Lemma 2 so that " $y \in V$ " in their conclusions is actually " $y \in$ $V \cap D$ ". Then we use this in Lemma 6 so that we have " $y_{t} \in Y_{t} \cap D$ " rather than just " $y_{t} \in Y_{t} \cap Y$ ". Finally, note that the only place in Lemma 6 where the Lindelöf property was used was in showing that $\left\{\left.f(x)\right|_{E}: x \in K\right\}$ is Lindelöf in $C_{p}(\bar{E})$ for some countable relatively compact subset $E$ of $Y$.

Corollary 1. Suppose that $X$ is Lindelöf and $\alpha$-favourable and $Y$ is Lindelöf and Cech-complete. Then for each separately continuous function $f: X \times Y \rightarrow \mathbb{R}$ there exists a residual set $R$ in $X$ such that $f$ is jointly continuous at each point of $R \times Y$.

Proof. Consider the mapping $\hat{f}: X \rightarrow C_{p}(Y)$ defined by $\hat{f}(x)(y):=f(x, y)$. Since $f$ is separately continuous, $\hat{f}: X \rightarrow C_{p}(Y)$ is continuous and hence $\hat{f}(X)$ is Lindelöf. The result now follows from Theorem 1 .

Corollary 2. Suppose that $X$ is separable and $\alpha$-favourable and $Y$ is Lindelöf and Čech-complete. Then for each separately continuous function $f: X \times Y \rightarrow \mathbb{R}$ there exists a residual set $R$ in $X$ such that $f$ is jointly continuous at each point of $R \times Y$. 
Proof. Consider the mapping $\hat{f}: X \rightarrow C_{p}(Y)$ defined by $\hat{f}(x)(y):=f(x, y)$. Since $f$ is separately continuous, $\hat{f}: X \rightarrow C_{p}(Y)$ is continuous and hence $\hat{f}(X)$ is separable in $C_{p}(Y)$. Thus for each compact subset $K$ of $Y,\left\{\left.f(x)\right|_{K}: x \in X\right\}$ is separable in $\left(C(K),\|\cdot\|_{\infty}\right)$ and so Lindelöf in $C_{p}(K)$. The result now follows from the Remark.

Corollary 3. For a non-empty closed and bounded convex subset $C$ of a weakly Lindelöf Banach space $X$, the following properties are equivalent:

(i) $(C$, weak) is $\alpha$-favourable;

(ii) $(C$, weak) is almost Čech-complete, i.e., contains as a dense subset a Čechcomplete space;

(iii) the points of continuity of $(C$, weak $)$ are a dense $G_{\delta}$ subset of $(C$, weak);

(iv) each non-empty convex relatively weak open subset of $C$ possesses nonempty relatively weak open subsets of arbitrarily small diameter.

Proof. The fact that (ii), (iii) and (iv) are equivalent follows from Theorem 3.1 in [10] and it is easy to see that (ii) implies (i). We shall complete the proof by showing that (i) implies (iii). To this end, let $C$ be a non-empty closed and bounded convex subset of $X$. We will consider the continuous linear mapping $I:(C$, weak $) \rightarrow C_{p}\left(B_{X^{*}}\right.$, weak $\left.^{*}\right)$ defined by $I(x)\left(x^{*}\right):=x^{*}(x)$. By our hypothesis, $(C$, weak) is both Lindelöf and $\alpha$-favourable. Therefore by Theorem 1 there exists a residual subset $R$ of $C$ such that $I$ is jointly continuous at each point of $R \times B_{X^{*}}$. Since $\left(B_{X^{*}}\right.$, weak $\left.{ }^{*}\right)$ is compact this is equivalent to $I$ being norm continuous at the points of $R$. Then, since $I$ is an isometry, it follows that each point of $R$ is in fact a point of continuity of $(C$, weak).

Remark. Let us end this paper by mentioning that:

(i) in [15] there is an example of a separately continuous function $f: X \times Y \rightarrow$ $\mathbb{R}$ defined on the product of an $\alpha$-favourable space $X$ and a compact space $Y$ such that $\{x \in X: f$ is jointly continuous at each point of $\{x\} \times Y\}$ is empty;

(ii) in [14 p. 313] there is an example of a separately continuous function $f: X \times Y \rightarrow \mathbb{R}$ defined on the product of a compact metric space $X$ and a completely metrizable (and hence Cech-complete) space $Y$ such that $\{x \in X: f$ is jointly continuous at each point of $\{x\} \times Y\}$ is empty;

(iii) the function $f:[0,1] \times C_{p}([0,1]) \rightarrow \mathbb{R}$ defined by $f(t, g):=g(t)$ is separately continuous on $[0,1] \times C_{p}([0,1])$ but nowhere jointly continuous, despite the fact that $C_{p}([0,1])$ is analytic (and hence hereditarily Lindelöf);

(iv) in 12 there is an example (under additional set-theoretical assumptions) of a separately continuous mapping $f: X \times Y \rightarrow \mathbb{R}$ defined on the product of a Baire space $X$ and a compact space $Y$ such that $C_{p}(Y)$ is hereditarily Lindelöf but for which $\{x \in X: f$ is jointly continuous at each point of $\{x\} \times$ $Y$ \} is empty;

(v) $Y$ is Lindelöf and Cech-complete if and only if it is the pre-image of a Polish space under a perfect surjective mapping (see [5, p. 441] and [5, Corollary 3.7]);

(vi) a slight modification of the proof of Theorem 1 yields an affirmative answer to the first question raised in Remark 1 of 7]. Namely, in terms of the definitions given in [7, if $X$ is a Banach space and ( $X$, weak) is Lindelöf is $X$ a generic continuity space? 


\section{REFERENCES}

[1] R. Baire, Sur les founctions des variables réelles, Ann. Math. Pura Appl. 3 (1899), 1-122.

[2] W. W. Bledsoe, Neighborly functions, Proc. Amer. Math. Soc. 3 (1952), 114-115. MR0045791 $(13: 634 d)$

[3] B. Cascales, I. Namioka and G. Vera, The Lindelöf Property and Fragmentability, Proc. Amer. Math. Soc. 128 (2000), 3301-3309. MR1695167 (2001b:54024)

[4] M. J. Fabian, Gâteaux Differentiability of Convex Functions and Topology. Weak Asplund Spaces, Canadian Mathematical Society Series of Monographs and Advanced Texts. Wiley Interscience, New York, 1997. MR1461271 (98h:46009)

[5] G. Gruenhage, Generalized metric spaces. Handbook of set-theoretic topology, 423-501, NorthHollard, Amsterdam, 1984. MR0776629(86h:54038)

[6] P. S. Kenderov, I. S. Kortezov and W. B. Moors, Continuity points of quasi-continuous mappings, Topology Appl. 109 (2001), 321-346. MR.1807395 (2001m:54008)

[7] P. S. Kenderov and W. B. Moors, A dual differentiation space without an equivalent locally uniformly rotund norm, J. Austral. Math. Soc. Ser. A 77 (2004), 357-364. MR2099807 (2005h:46026)

[8] P. S. Kenderov and J. Orihuela, On a generic factorisation theorem, Mathematika 42 (1995), 56-66. MR:1346672 (96h:54014)

[9] S. Mercourakis and S. Negrepontis, Banach spaces and Topology II. Recent progress in general topology (Prague, 1991), 493-536, North-Holland, Amsterdam, 1992. MR1229137

[10] W. B. Moors, The relationship between Goldstine's theorem and the convex point of continuity property, J. Math. Anal. and Appl. 188 (1994), 819-832. MR1305488 (95k:46024)

[11] W. B. Moors and J. R. Giles, Generic continuity of minimal set-valued mappings, J. Austral. Math. Soc. (Series A) 63 (1997), 238-262. MR1475564 (98k:46076)

[12] I. Namioka and R. Pol, Mappings of Baire spaces into function spaces and Kadec renorming, Israel J. Math. 78 (1992), 1-20. MR1194955 (94f:46020)

[13] J. O. Oxtoby, The Banach-Mazur game and Banach Category Theorem, in Contributions to the Theory of Games, vol 111, Annals of Math. Studies 39, Princeton, N. J. (1957), 158-163.

[14] Z. Piotrowski, Separate and joint continuity, Real Anal. Exchange 11 (1985/86), 293-322. MR.0844254 (87i:01042)

[15] M. Talagrand, Deux généralisations d'un théorème de I. Namioka, Pacific J. Math. 81 (1979), 239-251. MR0543747 (80k:54018)

Department of Mathematics, The University of Auckland, Private Bag 92019, AuckLAND, NeW ZEALAND

Current address: Institute of Mathematics, Bulgarian Academy of Science, Acad G. Bonchev Street, Block 8, 1113 Sofia, Bulgaria

E-mail address: pkend@math.bas.bg

E-mail address: vorednek@yahoo.com

Department of Mathematics, The University of Auckland, Private Bag 92019, AuckLAND, NEW ZEALAND

E-mail address: moors@math.auckland.ac.nz 